\title{
Vacuum Fluctuations in Optical Metamaterials Containing Nonlinear Dielectrics
}

\author{
Z. JAKŠIĆ ${ }^{a, *}$, S. Ostojić ${ }^{b}$, D. TAnASKoviĆ ${ }^{a}$ And J. MatoviĆc \\ ${ }^{a}$ Institute of Microelectronic Technologies and Single Crystals \\ IHTM - Institute of Chemistry, Technology and Metallurgy, University of Belgrade, Belgrade, Serbia \\ ${ }^{b}$ Faculty of Technology and Metallurgy, Belgrade, Serbia \\ ${ }^{c}$ Institute for Sensor and Actuator Systems ISAS \\ Vienna University of Technology, Vienna, Austria
}

\begin{abstract}
We investigated the influence of zero-point fluctuations (vacuum fluctuations, optical quantum noise) to the optical response of electromagnetic metamaterials containing dielectrics with third-order Kerr-like nonlinearity. We determined the zero-point noise and calculated it for different analytes, including those used in forensic analysis and organic pollutants. The zero-point noise level is highest for shortest-wavelength plasmons and decreases towards long-range plasmons. It may be tailored through a convenient design of the metamaterial structure. Since noise spectral power is proportional to the nonlinearity of the analyte species present, we considered the possibility to use zero-point noise as an auxiliary tool for identification of targeted nonlinear samples. We believe that our investigation could be of importance in homeland defence, forensics, biomedicine, etc.
\end{abstract}

PACS numbers: 73.20.Mf, 81.05.Xj, 42.50.Lc, 78.67.-n, 78.67.Pt

\section{Introduction}

Plasmonic devices belong to the most sensitive sensors of chemical or biological analytes [1], their conventional and widely used version being the surface plasmon resonance (SPR) devices. Metamaterial (MM) sensors [2] represent a step further in the same direction. We use here the term "metamaterial" in its general sense, i.e. to denote any structure furnishing electromagnetic response not readily found in Nature, and not only negative effective refractive index structures.

It is known that fluctuations and noise are the ultimate limiting factor of any sensing device. In SPR/MM sensors one encounters extrinsic sources (interrogating light beam fluctuations, noise of the readout detector and of the circuitry [1]). Intrinsic sources of noise include adsorption-desorption noise, optical thermal and flicker noise [3]. A component which may be very important in MM sensors is quantum noise, caused by zero-point energy fluctuations of the surface plasmon vacuum [4]. These fluctuations are especially pronounced near the metal-dielectric interface, exactly in the point of maximum sensitivity of such sensors. If the analyte material exhibits a Kerr-like optical nonlinearity, its refractive index will vary proportionally to the equivalent zero-point field, with an amplitude defined by the nonlinear susceptibility of the analyte [5].

In this paper we analyze the zero-point noise in metamaterial-based structures due to the Kerr nonlinearity of the analyte. We consider the applicability of its increase with rising nonlinear susceptibility to improve

* corresponding author; e-mail: jaksa@nanosys.ihtm.bg.ac.rs the nonlinear analyte recognition and boost the sensor selectivity.

\section{Theory}

We consider a simple flat slab of metamaterial onto which a thin layer of the analyte to be detected is adsorbed. An interrogating light beam is incident to the surface under an oblique angle and is coupled to the plasmon modes through the attenuated total reflection mechanism (a conventional Kretschmann configuration) [1]. The presence of the analyte changes the refractive index at the surface. Upon reflection an angular shift is detected in the reflected beam, and the amount of the shift is proportional to the refractive index difference between the analyte and the surroundings.

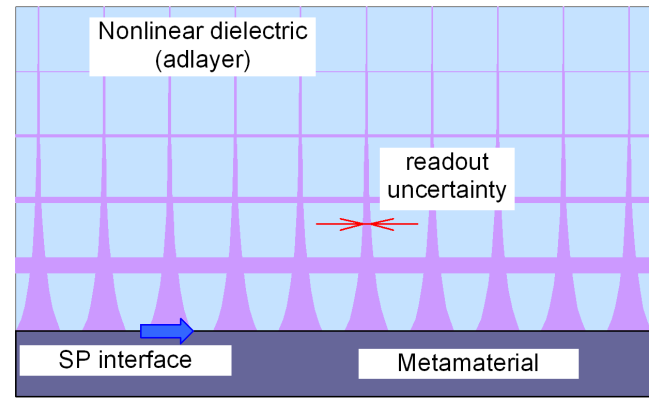

Fig. 1. Influence of zero-point fluctuations to dielectric permittivity in presence of nonlinear analyte. The line width corresponds to readout uncertainty.

We consider noise generated by the zero-point fluctuations of the vacuum field, which are the cause of 


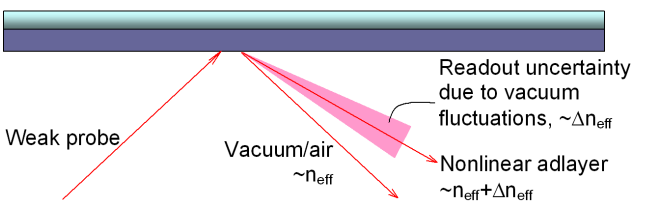

Fig. 2. "Heated highway mirage effect" of zero-point fluctuations.

spontaneous radiation and the appearance of CasimirPolder and London-van der Waals forces [6]. Zero-point field is most strongly felt near the surface of the MM sensor. Since the refractive index of an optically nonlinear material depends on the intensity of the electric field, zero-point fluctuations will directly cause fluctuations of the refractive index, Figs. 1, 2. This was described by Smolyaninov as the "heated highway mirage effect" [7]. It is connected with the quantum behavior of the plasmon states and does not depend on the number of photons in the system, if the interrogating beam intensity does not excite nonlinear effects. Since plasmons enhance the fields for several orders of magnitude [8], this condition is readily satisfied.

We are interested in the frequency range near the resonant frequency $\omega_{\mathrm{sp}}$ of the surface plasmon sensor, since this is the operating point of the device. At the same time, due to the existence of resonant enhancement, it will contain the most part of the contribution to the zero-point field.

The total zero-point energy is infinite and can be written as

$$
U=\frac{1}{2} \sum_{k}^{k_{\max }} \hbar \omega
$$

where $k$ includes all possible modes. This value at the sensor active area becomes finite if summation is cut off at the maximum wave vector, $k_{\max }$. Further, since the operating frequency $\omega=\omega_{\mathrm{sp}}$

$$
U=\frac{1}{2} \sum_{k}^{k_{\max }} \hbar \omega=\frac{1}{2} \sum_{k}^{k_{\max }} \hbar \omega_{\mathrm{sp}}=N_{\bmod } \hbar \omega_{\mathrm{sp}},
$$

where $N_{\text {mod }}$ is the number of modes per active area and $k_{\max }=1 / \lambda_{\min }$ is the maximum wave vector [9]. In real situations $\lambda_{\min }$ is typically $\approx 30 \mathrm{~nm}$. is

The number of modes on the top side of the active area

$$
N_{\text {mod }}=2 k_{\text {max }}^{2} a^{2} / \pi^{2},
$$

where $a$ is the side of the square active area. The total zero-point energy is

$$
U=\frac{\left\langle E_{\mathrm{sp}}^{2}\right\rangle}{4 \pi} a^{2}\left\langle\frac{1}{k_{\mathrm{sp}}}\right\rangle=\frac{\left\langle E_{\mathrm{sp}}^{2}\right\rangle}{4 \pi} a^{2}\left\langle\frac{1}{k_{\max }}\right\rangle .
$$

From (2), (3) and (4) the mean electric field of SP vacuum is

$$
\left\langle E_{\mathrm{sp}}^{2}\right\rangle=\frac{c^{2}}{10^{4} \pi} \hbar \omega_{\mathrm{sp}} k_{\max }^{3} .
$$

The refractive index at the SP interface is [10]:

$$
\begin{aligned}
n_{d} & =n_{d 0}+\Delta n_{d}=n_{d 0}+2 \frac{3}{4 n_{d 0}} \chi^{(3)}\left\langle E_{\mathrm{sp}}^{2}\right\rangle \\
& =n_{d 0}+2 n_{2}\left\langle E_{\mathrm{sp}}^{2}\right\rangle,
\end{aligned}
$$

where $n_{2}$ is the nonlinear refractive index coefficient, and $\chi^{(3)}$ is the third-order nonlinear susceptibility. One should note that there is a discrepancy between various literature sources in notation for nonlinear refractive index. We adopted here the one used in [10]. Also one should mind that in SI units the $\chi^{(3)}$ will be expressed in $\mathrm{m}^{2} / \mathrm{V}^{2}$ instead of the usual esu units; thus $n_{2}[\mathrm{SI}]=$ $(1 / 3)^{2} \times 10^{-8} n_{2}$ [esu], $\chi^{(3)}[\mathrm{SI}]=(4 \pi / 3)^{2} \times 10^{-8} \chi^{(3)}[\mathrm{esu}]$.

For a non-magnetic adlayer

$$
\begin{aligned}
& n_{d}=n_{d 0}+\Delta n_{d}=\sqrt{\varepsilon_{d} \mu_{d}}=\sqrt{\varepsilon_{d}}, \quad n_{d 0}^{2}=\varepsilon_{d 0}, \\
& \varepsilon_{d} \approx \varepsilon_{d 0}+2 \Delta n_{d} n_{d 0}, \\
& \Delta \varepsilon_{d}=4 \pi \chi^{(3)}\left\langle E_{\mathrm{sp}}^{2}\right\rangle=16 \chi^{(3)} \hbar \omega_{\mathrm{sp}} k_{\max }^{3},
\end{aligned}
$$

where $\omega_{\mathrm{sp}}$ is the surface plasmon resonance frequency [11]:

$$
\omega_{\mathrm{sp}}=\frac{\omega_{\mathrm{p}}}{\sqrt{1+\varepsilon_{d}}}, \quad \text { where } \quad \omega_{\mathrm{p}}=\sqrt{\frac{n_{\mathrm{e}} \mathrm{e}^{2}}{\varepsilon_{0} m_{\mathrm{e}}}} .
$$

Since $\varepsilon_{d}$ depends on $\omega_{\mathrm{sp}}$, Eq. (10) may be solved for $\omega_{\mathrm{sp}}$ as

$$
\begin{aligned}
& \omega_{\mathrm{sp}}=-\frac{1+\varepsilon_{d 0}}{3 C}+\left[2^{1 / 3}\left(1+\varepsilon_{d 0}\right)^{2}\right] /\left[3 C \left(-2-6 \varepsilon_{d 0}-6 \varepsilon_{d 0}^{2}-2 \varepsilon_{d 0}^{3}+27 C^{2} \omega_{\mathrm{p}}^{2}\right.\right. \\
& \left.\left.+\sqrt{-4\left(1+\varepsilon_{d 0}\right)^{6}-\left(-2-6 \varepsilon_{d 0}-6 \varepsilon_{d 0}^{2}-2 \varepsilon_{d 0}^{3}+27 C^{2} \omega_{\mathrm{p}}^{2}\right)^{2}}\right)^{3}\right] \\
& +\frac{1}{3 \times 2^{1 / 3} C}\left[3 C \left(-2-6 \varepsilon_{d 0}-6 \varepsilon_{d 0}^{2}-2 \varepsilon_{d 0}^{3}+27 C^{2} \omega_{\mathrm{p}}^{2}\right.\right. \\
& \left.\left.+\sqrt{-4\left(1+\varepsilon_{d 0}\right)^{6}-\left(-2-6 \varepsilon_{d 0}-6 \varepsilon_{d 0}^{2}-2 \varepsilon_{d 0}^{3}+27 C^{2} \omega_{\mathrm{p}}^{2}\right)^{2}}\right)^{3}\right]
\end{aligned}
$$


where $C=16 \hbar k_{\max }^{3} \chi^{(3)}$. In the case of (nano)plasmonic structures the value of $\omega_{\mathrm{sp}}$ is tailorable to a range of values.

The propagation constant for the guided mode is [1]:

$$
\beta=\frac{\omega}{c} \sqrt{\frac{\varepsilon_{d} \varepsilon_{m}}{\varepsilon_{d}+\varepsilon_{m}}},
$$

where the relative dielectric permittivity of metal is expressed according to Drude free-electron model as

$$
\varepsilon_{m}=\varepsilon_{0}\left[1-\omega_{\mathrm{p}}^{2} /\left(\omega^{2}+\mathrm{i} \gamma \omega\right)\right] .
$$

For low losses $\left(\operatorname{Re} \varepsilon_{m} \gg \operatorname{Im} \varepsilon_{m}\right)$ this becomes [1]:

$$
\begin{aligned}
\beta & =\frac{\omega}{c} \sqrt{\frac{\operatorname{Re}\left(\varepsilon_{m}\right) \varepsilon_{d}}{\operatorname{Re}\left(\varepsilon_{m}\right)+\varepsilon_{d}}} \\
& +\mathrm{i} \frac{\omega}{c}\left(\frac{\operatorname{Re}\left(\varepsilon_{m}\right) \varepsilon_{d}}{\operatorname{Re}\left(\varepsilon_{m}\right)+\varepsilon_{d}}\right)^{\frac{3}{2}} \frac{\operatorname{Im}\left(\varepsilon_{m}\right)}{2\left[\operatorname{Re}\left(\varepsilon_{m}\right)\right]^{2}} .
\end{aligned}
$$

The effective refractive index at the interface in the case when no adsorbed analyte is present (only a semi-infinite superstrate with a relative dielectric permittivity $\left.\varepsilon_{d}\right)$ is further determined as

$$
n_{\text {eff }}=c \operatorname{Re}(\beta) / \omega \text {. }
$$

The change of the effective refractive index due to the presence of an ultrathin analyte adlayer is finally observed as

$$
\Delta n_{\mathrm{eff}}=\frac{2 \operatorname{Re}\left[\Gamma_{d} \beta^{3}\right]}{(\omega / c)^{3} n_{d}^{3}} h \Delta n_{d}, \quad \Gamma_{d}=\beta^{2}-\omega^{2} \varepsilon_{0} \varepsilon_{d} \mu_{0},
$$

where $h$ is the thickness of the adlayer. For low losses in the metal part

$$
\frac{\Delta n_{\mathrm{eff}}}{\Delta n}=\left(\frac{\operatorname{Re}\left(\varepsilon_{m}\right)}{\operatorname{Re}\left(\varepsilon_{m}\right)+n_{d}^{2}}\right)^{\frac{3}{2}} \frac{2 n_{d}^{2}}{\sqrt{-\operatorname{Re}\left(\varepsilon_{m}\right)-n_{d}^{2}}} h \frac{\omega}{c} .
$$

\section{Results}

Some polar fluids (including toxic and explosive substances) exhibit large electro-optic effect. These include nitrotoluene $\left(n_{2}=3 \times 10^{-20}\right.$ [SI], taggant for explosive detection), benzene (important environmental pollutant, $\left.n_{2}=6 \times 10^{-21}[\mathrm{SI}]\right)$, polythiophene $\left(n_{2}=3.4 \times\right.$ $\left.10^{-19}[\mathrm{SI}]\right)$, etc $\left.[12-16]\right)$. Refractive index fluctuations vs. wavelength for some of them are shown in Fig. 3.

\section{Conclusions}

The influence of nonlinearity of adsorbed analyte to the level of noise of a SPR or metamaterial sensor was analyzed. It is concluded that zero-point noise may significantly alter permittivity/refractive index readout of nonlinear analytes. The influence of zero-point fluctuations is larger at shorter operating wavelengths. Since different levels of zero-point noise correspond to different nonlinear analytes, the determination of the vacuum fluctuation levels may be used to promote the MM sensor selectivity towards nonlinear analytes. The two kinds of data for this purpose may be collected by the conventional Kretschmann readout configuration - the reflection angle of the probe beam and the beam fluctuation around its mean value.

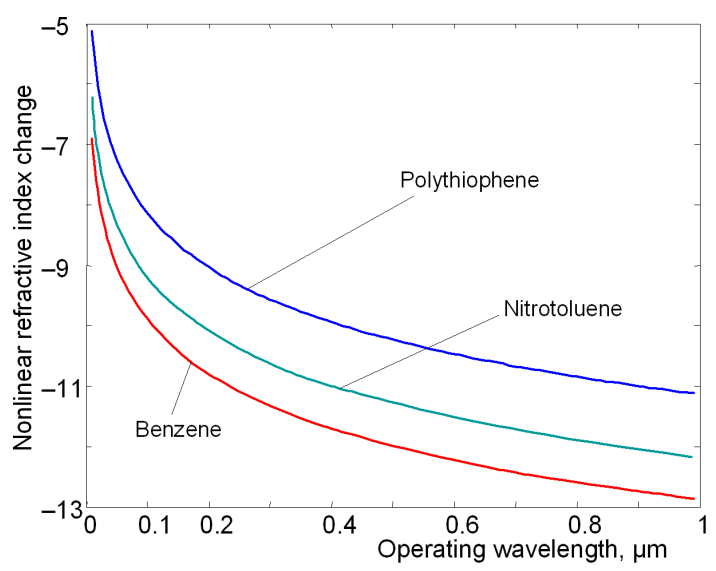

Fig. 3. Refractive index readout uncertainty due to zero-point fluctuations versus operating wavelength in various nonlinear materials.

\section{Acknowledgments}

This work was funded by the Austrian Science Fund (FWF) within the project L521 and by the Serbian Ministry of Science and Technology within the project 11027.

\section{References}

[1] Surface Plasmon Resonance Based Sensors, Ed. J. Homola, Springer, Berlin 2006.

[2] Z. Jakšić, O. Jakšić, Z. Djurić, C. Kment, J. Opt. A 9, S377 (2007).

[3] Z. Jakšić, O. Jakšić, J. Matović, J. Nanophot. 3, 031770 (2009).

[4] D.L. Mills, Nonlinear Optics - Basic Concepts, Springer, Berlin 1991.

[5] L. Mandel, E. Wolf, Optical Coherence and Quantum Optics, Cambridge University Press 1995.

[6] P.W. Milloni, The Quantum Vacuum, Academic Press, London 1994.

[7] I.I. Smolyaninov, Phys. Rev. Lett. 94, 057403 (2005).

[8] A. Zayats, I. Smolyaninov, A. Maradudin, Phys. Rep. 408, 131 (2005).

[9] A.G. Mal'shukov, Phys. Rep. 194, 343 (1990).

[10] R. Sutherland, Handbook of Nonlinear Optics, Marcel Dekker, New York 1996.

[11] S. Maier, Plasmonics: Fundamentals and Applications, Springer, Berlin 2007.

[12] P.P. Ho, R.R. Alfano, Phys. Rev. A 20, 2170 (1979).

[13] H. Hoshi, N. Nakamura, Y. Maruyama, T. Nakagawa, S. Suzuki, H. Shiromaru, Y. Achiba, Jpn. J. Appl. Phys. 30, L391 (1991).

[14] R. Dorsinville, L. Yang, R. Alfano, R. Zamboni, R. Danieli, G. Ruani, C. Taliani, Opt. Lett. 14, 1321 (1989).

[15] M. Sheik-Bahae, A.A. Said, T.-H. Wei, D.J. Hagan, E.W. Van Stiyland, IEEE J. Quant. Electron. 26, 760 (1990).

[16] T.D. Krauss, F.W. Wise, Appl. Phys. Lett. 65, 1739 (1994). 